\title{
Probing Interfacial and Surface Effects with Vibrational Electron Energy Loss Spectroscopy
}

\author{
Kartik Venkatraman ${ }^{1}$, Qianlang Liu ${ }^{1}$, Katia March ${ }^{2}$, Peter Rez $^{3}$ and Peter Crozier ${ }^{1}$ \\ 1. School for the Engineering of Matter, Transport and Energy, Arizona State University, Tempe, \\ Arizona 85287-6106, USA. \\ 2. LeRoy Eyring Center for Solid State Science, Arizona State University, Tempe, Arizona 85287-1704, \\ USA. \\ 3. Department of Physics, Arizona State University, Tempe, Arizona 85287-1704, USA.
}

The novel ability to detect vibrational excitations with monochromated electron energy-loss spectroscopy (EELS) has great potential in high spatial resolution characterization of materials [1]. Such instrumentation capabilities can be leveraged to investigate delocalized behavior of the bulk as well as localized behavior of surfaces and interfaces. For a comprehensive understanding of this technique, experiments need to be performed on simple model systems and the results need to be compared with theory. In this paper, we explore the experimental spatial variation in the vibrational stretch signals from the bulk, surfaces and interfaces when an electron beam is scanned across a $\mathrm{SiO}_{2} / \mathrm{Si}$ interface. The resultant profiles are interpreted in terms of non-relativistic and relativistic dielectric theories $[2,3]$.

A $3 \mu \mathrm{m}$ layer of $\mathrm{SiO}_{2}$ on a $\mathrm{Si}$ wafer was prepared for STEM EELS analysis by lifting out a focused ion beam (FIB) sample using a Nova 200 NanoLab (FEI) FIB. A NION UltraSTEM 100 aberrationcorrected electron microscope equipped with a monochromator was used to perform EELS linescans across the $\mathrm{SiO}_{2} / \mathrm{Si}$ interface. The microscope was operated at $60 \mathrm{kV}$, with probe convergence and collection semi-angles of 30 and $15 \mathrm{mrad}$ respectively. The data was processed for background subtraction and signal integration using the Gatan Microscopy Suite.

Figure 1a is a bright-field (BF) STEM image of the $\mathrm{Si} / \mathrm{SiO}_{2}$ interface that shows the position of the interface and the direction of the linescan. Typical background subtracted vibrational EELS spectra

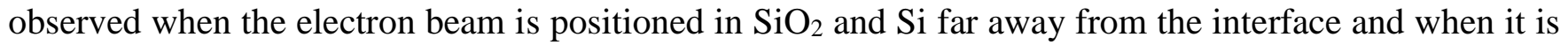
exactly at the $\mathrm{SiO}_{2} / \mathrm{Si}$ interface are shown in Figure 1b. The energy-loss spectrum shows a peak at 144 meV when the beam is in $\mathrm{SiO}_{2}$, which corresponds to the bulk $\mathrm{Si}-\mathrm{O}$ vibrational stretch. When the beam is outside the $\mathrm{SiO}_{2}$ in vacuum (aloof mode) the peak is located at $138 \mathrm{meV}$. When the probe is exactly at the $\mathrm{SiO}_{2} / \mathrm{Si}$ interface, the peak shifts to $136 \mathrm{meV}$ which corresponds to the $\mathrm{SiO}_{2} / \mathrm{Si}$ interfacial vibrational stretch. The signal drops to the background surface oxide level in Si.

The spatial variation of the bulk $\mathrm{Si}-\mathrm{O}$ vibrational stretch as a function of the distance from the $\mathrm{SiO}_{2} / \mathrm{Si}$ interface, is shown in Figure 2a. The integrated bulk Si-O vibrational signal starts to decrease with decreasing distance around $250 \mathrm{~nm}$ from the interface in $\mathrm{SiO}_{2}$ and drops to the background level right at the interface. The experimental profile is also compared to theoretical profiles based on non-relativistic and relativistic models of the classical dielectric theory in the figure [2,3]. The relativistic and nonrelativistic models are in agreement for $\mathrm{SiO}_{2}$. The relativistic model incorporates Ćerenkov losses and would be expected to give a better estimate of the intensity in Si. Figure $2 b$ shows the spatial variation of the $\mathrm{SiO}_{2} / \mathrm{Si}$ interfacial vibrational stretch with reference to that of the bulk signal. As the bulk vibrational stretch signal decreases in intensity in a fashion similar to Figure 2a, the interfacial stretch signal increases and peaks at the interface. The Begrenzung's effect causes the spatial variation in the bulk and 
interface signals to be correlated. The full width at half maximum of the interfacial signal is a couple of nanometers. We will discuss the spatial variation of the interfacial signal in Si and compare with detailed simulations of the experimental profiles considering Ćerenkov loss effects in $\mathrm{Si}$.

References:

[1] O.L. Krivanek et al., Nature, 514 (2014), p. 209.

[2] A. Howie and R.H. Milne, Ultramicroscopy, 18 (1985), p. 427.

[3] Z.L. Wang, Micron, 27 (1996), p. 265.

[4] The support from National Science Foundation CHE-1508667 and the use of TEM at John M.

Cowley Center at Arizona State University is gratefully acknowledged. Dr. Toshihiro Aoki is thanked for his help with acquiring data on the NION.

a)

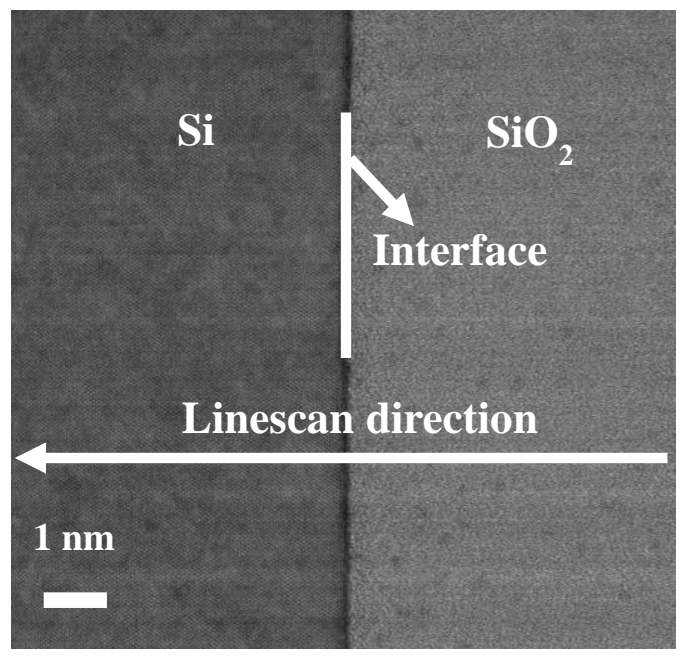

b)

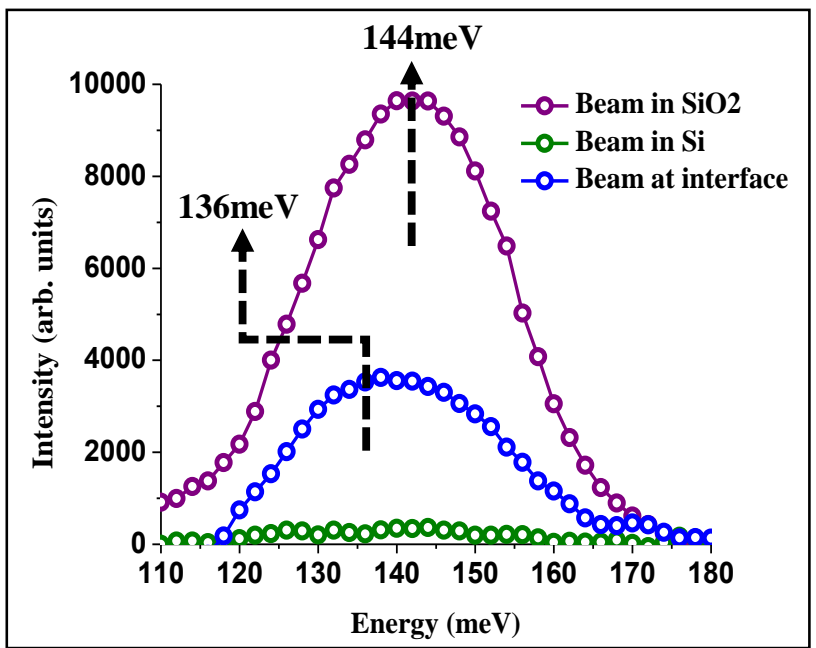

Figure 1. a) BF-STEM image of the $\mathrm{Si} / \mathrm{SiO}_{2}$ interface showing the direction of linescan. b) Typical vibrational spectra observed when the beam in far away from the interface in $\mathrm{SiO}_{2}$ and $\mathrm{Si}$ and when the beam is exactly at the interface.

a)

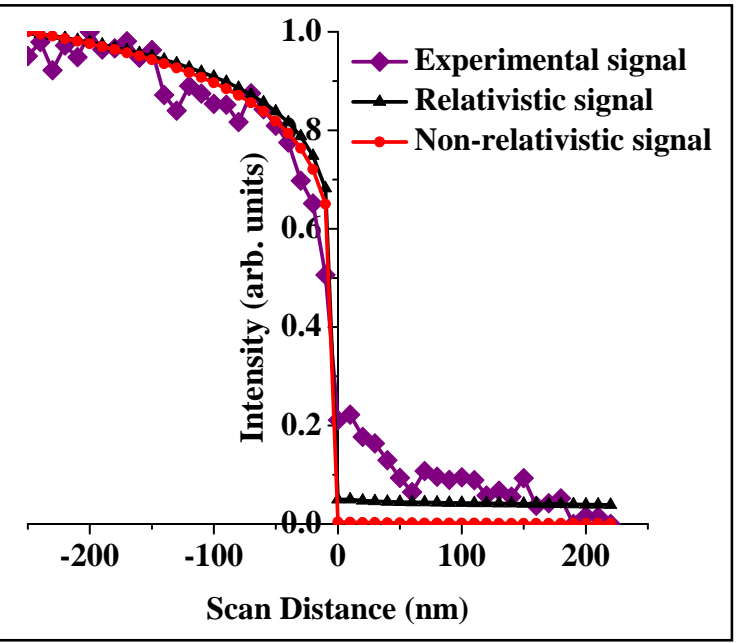

b)

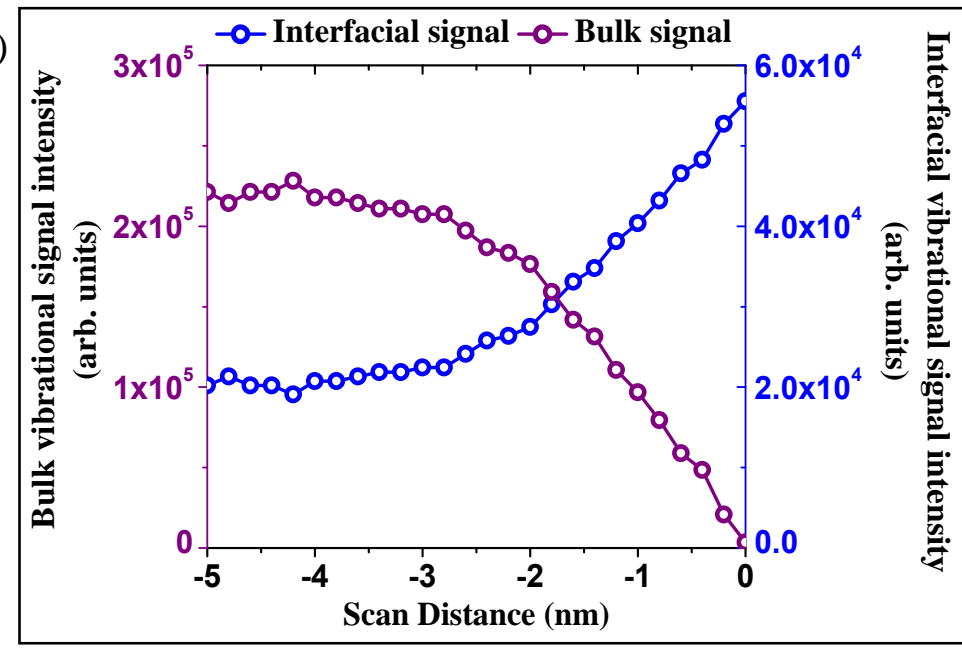

Figure 2. a) Experimental and theoretical agreement in the linescan profile for the bulk Si-O vibrational stretch. b) Comparison of the experimental linescan profile of the bulk $\mathrm{Si}-\mathrm{O}$ vibrational stretch with the $\mathrm{SiO}_{2} / \mathrm{Si}$ interfacial vibrational stretch when the beam is in $\mathrm{SiO}_{2}$. 